\title{
Late postpartum preeclampsia induced acute pulmonary edema, a rare presentation of a common disease: A case report
}

\author{
João Rua, ${ }^{1}$ Joana Costa, ${ }^{1}$ José Leite, ${ }^{1}$ Ricardo Marques, ${ }^{1}$ Joana Queiró, ${ }^{2}$ Jorge Fortuna, ${ }^{1}$ \\ Adriano Rodrigues ${ }^{1}$
}

${ }^{1}$ Internal Medicine Department B, General Hospital, University of Coimbra, Coimbra; ${ }^{2}$ Medical Emergency Unit, S. José Hospital, Central Lisboa Hospital Centre, Lisboa, Portugal

\begin{abstract}
Preeclampsia is a disorder of widespread vascular endothelial dysfunction and vasospasm associated with pregnancy. It can occur from the $20^{\text {th }}$ week to 4-6 weeks postpartum, resulting in hypertension, proteinuria and, in severe cases, end-organ damage and death. A 31-year-old puerpera enters the emergency room with complaints of dyspnea and leg edema, two weeks after the delivery. She was polipneic and hypertensive, had vascular congestion on thorax x-ray, elevated d-dimers and NT-proBNP. Meanwhile pulmonary embolism was suspected, she developed an acute pulmonary edema. Exams excluded embolism and cardiac abnormalities. New onset proteinuria allowed to diagnose a severe postpartum preeclampsia. About $5.7 \%$ of preeclampsia occurs in the postpartum but this is sometimes neglected. As progression to eclampsia isn't influenced by control of hypertension or severe symptoms and seizure complications are a major cause of morbimortality, its recognition and institution of prophylaxis with magnesium sulphate is crucial.
\end{abstract}

\section{Introduction}

Preeclampsia is a common disease of pregnancy, occurring in

Correspondence: João Rua, Internal Medicine Department B, General Hospital, University of Coimbra, Quinta dos Vales, São Martinho do Bispo 108, 3041-801 Coimbra, Portugal.

Tel.: +35.1914818022.

E-mail: Joaomfrua@gmail.com

Key words: Postpartum preeclampsia; Severe preeclampsia; Pulmonary edema.

Contributions: the authors contributed equally.

Conflict of interest: the authors declare no potential conflict of interest.

Funding: none.

Received for publication: 18 December 2017.

Revision received: 25 February 2018.

Accepted for publication: 12 March 2018.

This work is licensed under a Creative Commons Attribution 4.0 License (by-nc 4.0).

CCopyright J. Rua, 2018

Licensee PAGEPress, Italy

Emergency Care Journal 2018; 14:7240

doi:10.4081/ecj.2018.7240
$5-9 \%$ of all pregnancies, from the $20^{\text {th }}$ week of pregnancy to 6 weeks after delivery. It is characterized by widespread vascular endothelial dysfunction and vasospasm, resulting in new onset hypertension and proteinuria, commonly accompanied by edema, and can be complicated by life threatening features such as acute pulmonary edema, renal failure, hemolysis, elevated liver enzime levels, low platelet levels (HELLP) syndrome and eclampsia, being among the 4 leading causes of maternal death. Late postpartum preeclampsia is considered uncommon, and it is even rarer with severe features. We present a case of an acute pulmonary edema induced by a postpartum preeclampsia in a 31-year-old woman, occurring two weeks after delivery.

\section{Case Report}

A 31-year-old african woman was admitted at the emergency room of the maternal unit of our hospital complaining of fatigue, dyspnea, dry cough and leg swollening. Two weeks had passed since her delivery and the symptoms had started 5 days before the admission. She denied fever, chest pain or metrorrhagia. Her pathological priors were unremarkable, including 3 previous successful pregnancies without any relevant events, and she didn't take any medications previously to this pregnancy when she developed gestational diabetes. There were no other medical problems during pregnancy but the post-delivery period was complicated by abundant haemorrhage which prolonged her admission.

At admission she was polipneic with $\mathrm{satO}_{2} 90 \%$ breathing air. Her blood pressure (BP) was $151 / 89 \mathrm{~mm} \mathrm{Hg}$ and her heart rate (HR) was $72 \mathrm{bpm}$. She was pale but both cardiac and pulmonary sounds were normal, as was the abdominal and gynecologic exam. Moderate bilateral leg edema stood out. Oxygen was started, achieving $94 \% \mathrm{satO}_{2}$ with $27 \% \mathrm{fiO}_{2}$, and a thorax x-ray was taken, showing hilar ingurgitation and vascular congestion. Blood analysis showed a microcytic hypochromic anemia with $\mathrm{Hb} 8.1 \mathrm{gr} / \mathrm{dL}$, no leukocytosis or thrombocytopenia, normal renal and hepatic function, C-reactive protein of $5 \mathrm{mg} / \mathrm{dl}$ (normal $<1$ ) and d-dimers of $14361 \mathrm{ng} / \mathrm{mL}$ (normal<232). She was started on low-molecularweight heparin on $1 \mathrm{mg} / \mathrm{kg}$ dosing every $12 \mathrm{~h}$ and was transferred to the emergency room of the acute adult unit as a pulmonary embolism was highly suspected.

The journey was short but her clinical status worsened. At admission, she was again frankly polypneic, with 32 cycles per minute, with $\mathrm{satO}_{2}$ of $88 \%$ with $\mathrm{fiO}_{2} 35 \%$, presented mucous pink sputum and bilateral crackles in the lower half of both pulmonary fields. Her BP was now 163/96 mm Hg and HR $96 \mathrm{bpm}$. She was immediately started on furosemide $40 \mathrm{mg}$ iv and isosorbide dinitrate (ID) perfusion, titrated for a $25 \%$ BP reduction, as an acute pulmonary edema (APE) was diagnosed. A blood gas analysis showed hypoxemia of $62 \mathrm{~mm} \mathrm{Hg}$ and a new x-ray exposed marked bilateral congestion with alveolar opacities surrounding the hila in 
a full-blown butterfly wing pattern (Figure 1). Transthoracic echocardiogram showed normal ventricular function, without any valvular dysfunction or pericardial effusion and the thorax angioCT excluded pulmonary embolism and pneumonia. Serial electrocardiograms and myocardial necrosis markers also had no suggestion of an infarction. The NT-proBNP was $732 \mathrm{pg} / \mu \mathrm{L}$ $(\mathrm{N}<300)$.

The patient was steadily improving with stable BP under high dosis of isossorbid dinitrate, marked diuresis and decreasing oxygen needs. The cause of the APE was unclear. Although she presented with hypertension, hypertensive APE was thought unlikely considering both her blood pressure and her structurally normal heart. The result of the urinalysis showed considerable proteinuria, completing the typically described presentation of preeclampsia: new-onset hypertension, leg edema and proteinuria, and clarifying the diagnosis, presenting severely in the post-partum as APE. She was started on eclampsia prophylaxis with magnesium sulfate intravenous perfusion for $24 \mathrm{~h}$.

The patient felt some headaches but no seizures occurred and after $48 \mathrm{~h}$ the ID perfusion rate was gradually decreased, being altered to oral nifedipin $10 \mathrm{mg}$ twice daily at $72 \mathrm{~h}$. The patient mantained normal BP and was transferred to the maternal unit were she remained under surveillance for a few days before being discharged already with no need for anti-hypertensive medication. She was reevaluated several times in the next few months, remaining asymptomatic and without any evidence of arterial hypertension.

\section{Discussion}

Preeclampsia is a disorder of widespread vascular endothelial dysfunction and vasospasm associated with pregnancy, that results in hypertension, proteinuria and end-organ damage in severe cases. ${ }^{1}$ It is part of the spectrum of hypertensive diseases of pregnancy that also include chronic hypertension, gestational hypertension and chronic hypertension superimposed with preeclampsia. It affects about 5-9\% of all pregnancies and may occur from the 20th week of gestation to 4-6 weeks after delivery. ${ }^{2-4}$ In a 10 -year retrospective study, $5.7 \%$ of preeclampsia cases were diagnosed postpartum, with $14.7 \%$ of those progressing to eclampsia. ${ }^{5}$ Late postpartum preeclampsia, occurring more than $48 \mathrm{~h}$ after delivery is considered uncommon. ${ }^{4}$

The pathologic mechanism isn't fully understood but it is accepted that its pathogenesis is linked to abnormal placentation and placental development due to altered immunologic response in the fetal-maternal interphase. This leads to placental hypoperfusion and ischemia, generating high levels of soluble fms-like tyrosine kinase and endoglin, that interact with the vascular endothelium growth factor and the placental growth factor with detrimental consequences to the endothelial function leading to hypertension and proteinuria. The nitric oxide pathway is also affected due to low levels of its synthetase, resulting in vasoconstriction in the placental bed, kidneys and other organs vasculature, which in combination with augmented oxidative stress, results in microtrombi formation and ischemic phenomena. A systemic response reaction, with overexpression of placental type 4 toll-like receptors and inflamatory cytokines leads to edema and vascular extravasation. Finally, altered molecular structure of the glycocalix hyaluronic acid in the feto-maternal interface is responsible for intra-uterine growth retardation. ${ }^{3,6}$
The disease manifestations vary from mild to severe with sudden deterioration and multiple end-organ damage in the mother's heart, liver, kidney, hematopoietic and central nervous system, uterus, placenta and the fetus. ${ }^{3}$ There can be a myriad of symptoms, being headaches the most common. Shortness of breath, visual changes, nausea, vomiting, epigastric pain and edema are also possible. When severe, marked hypertension and end-organ damage become evident with pulmonary edema, oliguria, renal failure, impaired hepatic function or thrombocytopenia. ${ }^{4}$ Organ infarction or hemorrhage, congestive heart failure or pulmonary edema, renal failure and HELLP syndrome are potentially life threatening. ${ }^{7}$ Preeclampsia also leads to a reduction in plasma oncotic pressure, altered endothelial permeability and decrease in colloid osmotic pressure to left ventricular end-diastolic pressure gradient. Those changes interact with fluid volume changes and sympathetic hyperactivity vasoconstriction, causing increased ventricular afterload and redistribution from the peripheral circulation to the pulmonary vessels, leading to pulmonary edema as occurred with our patient. ${ }^{8}$ Sibai et al. describe a $2.9 \%$ occurrence of acute pulmonary edema in 1290 pregnancies complicated with severe preeclampsia, out of 270.000 deliveries in a 9-year spam, with a global incidence of $0.014 \%$. This was 4.2 times more common in patients with previous chronic hypertension than with pure preeclampsia and the average time of onset was $71 \mathrm{~h} .{ }^{9}$ Lower platelet count, increased serum uric acid and use of magnesium sulfate in the delivery process were the risk factors for pulmonary edema found by Shitah Ganshi in a 92 cohort of women. ${ }^{10}$

The diagnosis of preeclampsia (Table 1) requires a systolic BP of at least $140 \mathrm{~mm}$ or a diastolic BP of at least $90 \mathrm{~mm}$ on two occasions, at least 4 hours apart plus new onset proteinuria (defined by $300 \mathrm{mg} / 24 \mathrm{~h}$ or protein/creatinine ratio $\geq 0.3$ or 2 urine protein dipstick measurement $\geq 1+$ taken $6 \mathrm{~h}$ apart) or a severe feature which include an elevated creatinine level $(>1.1 \mathrm{mg} / \mathrm{dL})$, hepatic dysfunction (transaminase $>2 \times$ upper limit of normal values), new onset headaches or visual disturbances, platelet count of $<100 \times 10^{3} / \mathrm{L}$ or pulmonary edema. ${ }^{11}$

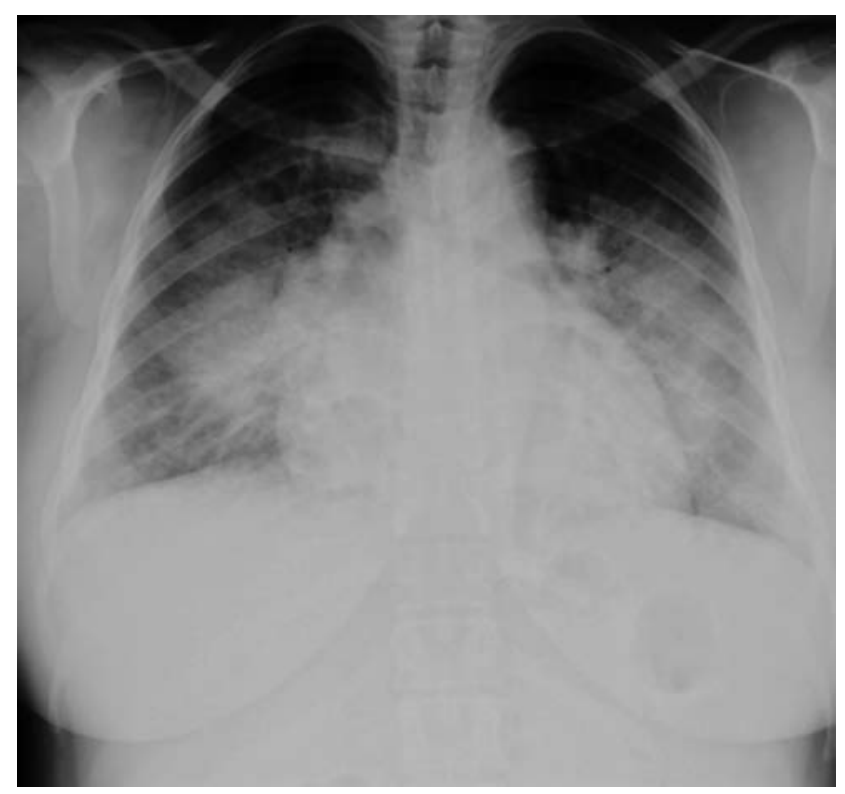

Figure 1. Thorax $x$-ray with butterfly wing pattern due to acute pulmonary edema. 
The occurrence of dyspnea in the postpartum has a myriad of possible causes and requires a meticulous differential diagnosis (Table 2). For diagnostic purposes, dyspnea causes can be grouped according to the absence or presence of pulmonary edema, with the latter subdivided in cardiogenic or non-cardiogenic etiology. In the non-pulmonary edema related conditions, pulmonary embolism, amniotic fluid embolism, pneumonia, septic respiratory dysfunction and aspiration of gastric content are the main causes. In the cardiogenic-APE group the most relevant are peripartum cardiomyopathy, preeclampsia-induced cardiomyopathy, underlying structural or valvular heart diseases and myocardial ischemia. Finally, the possible causes for non-cardiogenic APE are iatrogenic fluid overload, thyroid disease, drug-induced pulmonary edema, acute respiratory distress syndrome, preeclampsia-related APE and rheumathologic conditions as systemic lupus erythematosus and vasculitis. ${ }^{4}$ To distinguish these conditions, the clinical examination has major clues in the usual APE new onset crackles in both pulmonary fields, confirmed by thorax x-ray or CT, also used to exclude pneumonia and pulmonary embolism. Crucially important is also the echocardiogram that allows the evaluation of the heart morphology and function, establishing the type of APE. ${ }^{4}$ As for this case, non-awareness for the possibility of late postpartum preeclampsia by the emergency physicians, delayed the treatment and could have had important consequences, such as progression to eclampsia.

In the US, preeclampsia and eclampsia are among the 4 leading causes of maternal death, responsible for $10-15 \%$ of maternal deaths. ${ }^{4,10}$ Ideal methods for prevention, screening, diagnosis and management have not been identified due to incomplete understanding of the pathophysiology of preeclampsia. ${ }^{3}$

The main goal of treatment is always maternal safety, followed by delivering an healthy newborn. ${ }^{4}$ Obviously, in postpartum the focus of treatment is to prevent eclampsia and major complications of severe preeclampsia.

Magnesium sulfate should be administered as eclampsia prophylaxis, 4-6 grams diluted in $100 \mathrm{~mL}$ of neutral saline in $20 \mathrm{~min}$, followed by a 1-2 gram per hour perfusion during the $24 \mathrm{~h}$ after the diagnosis. As hypermagnesemia may cause significant toxicity, such as central nervous system depression, respiratory paralysis and cardiac arrest, routine magnesium serum concentration testing and clinical evaluation should be made every 6 hours, with great value of the presence of deep reflexes in reassuring of the absense of toxicity. ${ }^{7,11}$

Antihypertensive medications don't influence the progression to eclampsia but may avoid severe preeclampsia complications. The threshold for treatment is systolic $\mathrm{BP}>160 \mathrm{~mm} \mathrm{Hg}$ or diastolic BP above $100 \mathrm{~mm} \mathrm{Hg}$ in two separate occasions and the target will be for systolic blood pressure between 140-155 $\mathrm{mm} \mathrm{Hg}$ and diastolic blood pressure $90-105 \mathrm{~mm} \mathrm{Hg}$ to ensure an adequate placental perfusion. Recommended drugs are labetalol, nifedipine and hydralazine. ${ }^{3,4,8}$ In postpartum preeclampsia there is no recommended target BP but clinicians must be aware of the possible deleterious effects of aggressive BP lowering. ${ }^{4}$ In case of APE, as in our case report, the reduction of critically high blood pressure is urgent and decisive for a favorable outcome. Nitroglycerin is the drug of choice in preeclampsia-associated APE and it is given in iv infusion starting at $5 \mu \mathrm{g} / \mathrm{min}$, increased every 5 minutes until a maximum of $100 \mu \mathrm{g} / \mathrm{min}^{8}{ }^{8}$ Isosorbide dinitrate or sodium nitroprussiate perfusions, as well as loop diuretics may also be used., ${ }^{42}$ Labetalol is not recommended as its negative inotropic effects can be deleterious. ${ }^{8}$

In case of eminent respiratory failure, despite the risk of aspiration of gastric content, non-invasive mechanical ventilation should be attempted as it provides the advantages of mechanical ventilation without the complications of intubation of pregnant women who are hypertensive, such as intracerebral hemorrhage. ${ }^{8}$

Table 1. Preeclampsia diagnostic criteria.

\begin{tabular}{|c|c|c|}
\hline \multicolumn{3}{|c|}{$\begin{array}{l}\text { New onset systolic blood pressure of at least } 140 \mathrm{~mm} \text { or a diastolic } \\
\text { blood pressure of at least } 90 \mathrm{~mm} \text { on two occasions, at least } 4 \text { hours apart }\end{array}$} \\
\hline \multicolumn{3}{|c|}{ PLUS } \\
\hline New onset proteinuria & or & One severe feature \\
\hline $300 \mathrm{mg}$ on $24 \mathrm{~h}$ measurement & & Elevated creatinine level (>1.1mg/dL) \\
\hline Protein/creatinine ratio $\geq 0.3$ & & Hepatic dysfunction (transaminase $>2 \times$ upper limit of normal values) \\
\hline \multirow[t]{3}{*}{2 urine protein dipstick measurement $\geq 1+$ taken $6 \mathrm{~h}$ apart } & & New onset headaches or visual disturbances \\
\hline & & Platelet count of $<100 \times 10^{3} / \mathrm{L}$ \\
\hline & & Pulmonary edema \\
\hline
\end{tabular}

Table 2. Differential diagnosis of dyspnea in the postpartum.

\begin{tabular}{lll} 
Non-pulmonary edema & \multicolumn{2}{c}{ Pulmonary edema } \\
\hline Aspiration of gastric content & Cardiogenic & Non-cardiogenic \\
Amniotic fluid embolism & Myocardial ischemia & Acute respiratory distress syndrome \\
Pneumonia & Structural heart disease & Drug induced \\
Pulmonary embolism & Valvular heart disease & Iartrogenic fluid overload \\
Septic respiratory dysfunction & Peripartum cardiomyopathy & Preeclampsia induced \\
& & Thyroid disease \\
\hline
\end{tabular}




\section{Conclusions}

As exposed, early suspicion and treatment are of focal importance, which can be jeopardized by the emergency physicians lowawareness for this rare complication of the postpartum. As the progression to eclampsia is not influenced by adequate control of hypertension or other severe symptoms and complications related to seizures are a major cause of death and morbility in this population, recognition and institution of prophylaxis with magnesium sulphate perfusion is also crucial to a good outcome. ${ }^{2}$

\section{References}

1. Lim K, Steinberg G, Ramus RM. Preeclampsia. Available from: Medscape.com. Accessed: 2017.

2. Yancey LM, Withers E, Bakes K, Abbott J. Postpartum Preeclampsia: Emergency Department presentation and management. J Emerg Med 2011;40:380-4.

3. Rosser ML, Katz NT. Preeclampsia: an Obstetrician's Perspective. Adv Chronic Kidney Dis 2013;20:287-96.

4. Prueksaritanond S, Ali AM, Aronu GM, et al. An uncommon cause of shortness of breath in a young puerpera. Case Rep
Obstet Gynecol 2013;710620.

5. Matthys LA, Coppage KH, Lambers DS, et al. Delayed postpartum preeclampsia: An experience of 151 cases. Am J Obstet Gynecol 2004;190:1464-6.

6. E-Sayed AAF. Preeclampsia: A review of the pathogenesis and possible management strategies based on its pathophysiological derangements. Taiwan J Obstet Gynecol 2017;56:593-8.

7. Sibai BM. Etiology and management of postpartum hypertension-preeclampsia. Am J Obstet Gynecol 2012;206:470-5.

8. Dennis AT, Solnordal CB. Acute pulmonary oedema in pregnant women. Anaesthesia 2012;67:646-59.

9. Sibai BM, Mabie BC, Harvey CJ, Gonzalez AR. Pulmonary edema in severe preeclampsia-ecclampsia: analysis of thirtyseven consecutive cases. Am J Obstet Ginecol 1987;156:11749.

10. Gandhi S, Sun D, Park AL, et al. The Pulmonary Edema Preeclampsia Evaluation (PEPE) Study. J Obstet Gynaecol Can 2014;36:1065-70.

11. Leeman L, Dresang LT, Fontaine P. Hypertensive disorders of Pregnancy. Am Fam Physician 2016;93:121-7.

12. Cunningham C, Rivera J, Spence D. Severe preeclampsia, pulmonary edema and peripartum cardiomyopathy in a primigravida patient. AANA J 2011;79:249-55. 